\title{
ON TURÁN TYPE INEQUALITIES FOR MODIFIED BESSEL FUNCTIONS
}

\author{
ÁRPÁD BARICZ AND SAMINATHAN PONNUSAMY
}

(Communicated by Sergei K. Suslov)

\begin{abstract}
In this note our aim is to point out that certain inequalities for modified Bessel functions of the first and second kind, deduced recently by Laforgia and Natalini, are in fact equivalent to the corresponding Turán type inequalities for these functions. Moreover, we present some new Turán type inequalities for the aforementioned functions and we show that their product is decreasing as a function of the order, which has an application in the study of stability of radially symmetric solutions in a generalized FitzHugh-Nagumo equation in two spatial dimensions. At the end of this note an open problem is posed, which may be of interest for further research.
\end{abstract}

\section{Some inequalities For modified Bessel FunCtions}

Let us denote with $I_{\nu}$ and $K_{\nu}$ the modified Bessel functions of the first and second kind, respectively. For definitions, recurrence formulas and other properties of modified Bessel functions of the first and second kind we refer to the classical book of Watson 35].

In 2007, motivated by a problem which arises in biophysics, Penfold et al. 31 proved that the product of the modified Bessel functions of the first and second kind, i.e. $u \mapsto P_{\nu}(u)=I_{\nu}(u) K_{\nu}(u)$, is strictly decreasing on $(0, \infty)$ for all $\nu \geq 0$. It is worth mentioning that this result for $\nu=n \geq 1$, a positive integer, was verified in 1950 by Phillips and Malin [32. In order to shorten the proof due to Penfold et al. 31, recently the first author [5] pointed out that the Turán type inequalities for modified Bessel functions of the first and second kinds are in fact equivalent to some known inequalities for the logarithmic derivatives of the functions in question. For the reader's convenience we recall here the historical facts for these Turán type inequalities (see [5, 8] for more details). More precisely, in view of the recurrence relations

$$
I_{\nu-1}(u)=(\nu / u) I_{\nu}(u)+I_{\nu}^{\prime}(u)
$$

and

$$
I_{\nu+1}(u)=I_{\nu}^{\prime}(u)-(\nu / u) I_{\nu}(u)
$$

the Turán type inequality

$$
I_{\nu-1}(u) I_{\nu+1}(u)-\left[I_{\nu}(u)\right]^{2}<0
$$

Received by the editors April 23, 2010 and, in revised form, June 28, 2011.

2010 Mathematics Subject Classification. Primary 33C10, 39B62.

Key words and phrases. Modified Bessel functions, Turán-type inequalities, completely monotonic functions. 
is equivalent to

$$
u I_{\nu}^{\prime}(u) / I_{\nu}(u)<\sqrt{u^{2}+\nu^{2}},
$$

where $\nu>-1$ and $u>0$. To the best of the authors' knowledge the Turán type inequality (2) for $\nu>-1$ was proved first in 1951 by Thiruvenkatachar and Nanjundiah 34 and later in 1974 by Amos [1, p. 243] for $\nu \geq 0$. In 1991 Joshi and Bissu [24] proved also (2) for $\nu \geq 0$, while in 1994 Lorch [27] proved that (2) in fact holds for all $\nu \geq-1 / 2$ and $u>0$. Recently, the first author 8 reconsidered the proof of Joshi and Bissu 24] and pointed out that (2) and (3) hold true for all $\nu>-1$ and that the constant zero on the right-hand side of (2) is the best possible. It is worth mentioning that in fact the function $\nu \mapsto I_{\nu}(u)$ is log-concave on $(-1, \infty)$ for each fixed $u>0$, as was pointed out by Baricz [7. Finally, we note that the inequality (3) for $\nu>0$ was first proved by Gronwall [13 in 1932, motivated by a problem in wave mechanics. This inequality in 1950 appeared also in Phillips and Malin's paper [32 for $\nu=n \geq 1$, a positive integer.

Similarly, by using the recurrence relations

$$
K_{\nu-1}(u)=-(\nu / u) K_{\nu}(u)-K_{\nu}^{\prime}(u)
$$

and

$$
K_{\nu+1}(u)=-K_{\nu}^{\prime}(u)+(\nu / u) K_{\nu}(u),
$$

it is easy to prove (see [5, 8]) that the Turán type inequality

$$
K_{\nu-1}(u) K_{\nu+1}(u)-\left[K_{\nu}(u)\right]^{2}>0
$$

is equivalent to

$$
u K_{\nu}^{\prime}(u) / K_{\nu}(u)<-\sqrt{u^{2}+\nu^{2}},
$$

where $\nu \in \mathbb{R}$ and $u>0$. The Turán type inequality (6) was proved in 1978 by Ismail and Muldoon [23 and recently by the first author (see [7, 8]) by using different methods. The constant zero on the right-hand side of (6) is the best possible. Note that for $\nu>1 / 2$ the Turán type inequality (6) appears also on Laforgia and Natalini's paper [25. It is also worth mentioning here that Ismail and Muldoon 23 , by using the Nicholson formula concerning the product of two modified Bessel functions of different order, proved that 23 the function $\nu \mapsto K_{\nu+a}(u) / K_{\nu}(u)$ is increasing on $\mathbb{R}$ for each fixed $u>0$ and $a>0$. As Muldoon 30] pointed out, this implies that $\nu \mapsto K_{\nu}(u)$ is log-convex on $\mathbb{R}$ for each fixed $u>0$. Recently, by using the Hölder-Rogers inequality, the first author [7] pointed out that the function $\nu \mapsto K_{\nu}(u)$ is in fact strictly log-convex on $\mathbb{R}$ for each fixed $u>0$. Finally, we note that the inequality (7) was proved for $\nu=n \geq 1$, a positive integer, by Phillips and Malin 32 in 1950.

Recently, motivated by some applications in finite elasticity, Laforgia and Natalini [26] proved the following inequalities:

$$
\frac{I_{\nu}(u)}{I_{\nu-1}(u)}>\frac{-\nu+\sqrt{u^{2}+\nu^{2}}}{u}
$$

and

$$
\frac{K_{\nu}(u)}{K_{\nu-1}(u)}<\frac{\nu+\sqrt{u^{2}+\nu^{2}}}{u},
$$


where $u>0$ and $\nu \geq 0$ in the first inequality, and $u>0$ and $\nu \in \mathbb{R}$ in the second inequality. However, inequality (8) is not new; as far as we know it was proved first by Amos [1 in 1974. It is important to note here that Laforgia and Natalini in order to deduce (8) and (9) used the Turán type inequalities (2) and (6). Moreover, inequality (8) improves a recently deduced inequality by the first author [6, which is useful in the study of the generalized Marcum $Q$-function applied frequently in radar signal processing. See [6, 26] for more details and also [2, 3, 4, 7, 8, 24, 25, 27, 34 for more details on Turán type inequalities.

Here our aim is to show that the inequalities (8) and (9) are in fact equivalent to the corresponding Turán type inequalities (2) and (6). To see this we just need to rewrite the inequality (3), by using (11), in the following form:

$$
\frac{I_{\nu-1}(u)}{I_{\nu}(u)}<\frac{\nu+\sqrt{u^{2}+\nu^{2}}}{u},
$$

which is actually equivalent to (8). Thus, the inequalities (2), (3) and (8) are equivalent and hold true for all $\nu>-1$ and $u>0$. Similarly, in view of (4), the inequality (7) is equivalent to

$$
\frac{K_{\nu-1}(u)}{K_{\nu}(u)}>\frac{-\nu+\sqrt{u^{2}+\nu^{2}}}{u},
$$

which is in fact equivalent to (9). Thus, the inequalities (6), (7) and (9) are equivalent.

Moreover, by using the corresponding counterparts of the Turán type inequalities (2) and (6) (or inequalities (3) and (7)), we can obtain in a similar way the corresponding counterparts of inequalities (8) and (9). More precisely, consider the Turán type inequalities

$$
I_{\nu}^{2}(u)-I_{\nu-1}(u) I_{\nu+1}(u)<I_{\nu}^{2}(u) /(\nu+1)
$$

and

$$
K_{\nu}^{2}(u)-K_{\nu-1}(u) K_{\nu+1}(u)>K_{\nu}^{2}(u) /(1-\nu) .
$$

Inequality (10), which holds for all $\nu>-1$ and $u>0$, was proved using different arguments in 1951 by Thiruvenkatachar and Nanjundiah 34 for $\nu>-1$, in 1991 by Joshi and Bissu [24 for $\nu \geq 0$, and recently by the first author [4, 8] for $\nu>-1$. Note that the constant $1 /(\nu+1)$ on the right-hand side of (10) is the best possible, as was pointed out recently in [8]. The Turán type inequality (11), which holds for all $\nu>1$ and $u>0$, was proved recently in [8. We note that the constant $1 /(1-\nu)$ on the right-hand side of (11) is the best possible.

Now, consider the corresponding counterparts of (3) and (7), namely

$$
u I_{\nu}^{\prime}(u) / I_{\nu}(u)>\sqrt{u^{2} \nu /(\nu+1)+\nu^{2}}
$$

and

$$
u K_{\nu}^{\prime}(u) / K_{\nu}(u)>-\sqrt{u^{2} \nu /(\nu-1)+\nu^{2}} .
$$

The inequality (12) is equivalent to (10) and holds true for all $\nu>-1$ and $u>0$, as was pointed out in [5]. This inequality appears also in Phillips and Malin's paper 32 for $\nu=n \geq 1$, a positive integer. The inequality (13) is valid for all $\nu>1$ and $u>0$. For $\nu=n>1$, a positive integer, it was proved by Phillips and Malin [32, and for $\nu>1$ by the first author [8]. Note that (13) is equivalent to (11), which can 
be verified by using the corresponding recurrence relations for the modified Bessel function of the second kind, mentioned above.

Now, our aim is to show that by using the same argument as in the proof of (8) and (9) we can obtain easily the counterparts of (8) and (9). Namely, by using (19) we conclude that (10) or (12) is equivalent to

$$
\frac{I_{\nu}(u)}{I_{\nu-1}(u)}<\frac{-\nu+\sqrt{\frac{\nu}{\nu+1} u^{2}+\nu^{2}}}{\frac{\nu}{\nu+1} u},
$$

which holds for all $u>0$ and all $\nu>0$. Similarly, in view of (44), we conclude that (11) or (13) is equivalent to

$$
\frac{K_{\nu}(u)}{K_{\nu-1}(u)}>\frac{\nu+\sqrt{\frac{\nu}{\nu-1} u^{2}+\nu^{2}}}{\frac{\nu}{\nu-1} u},
$$

which holds for all $\nu>1$ and all $u>0$. We note that inequalities (14) and (15) in the above forms seem to be new.

Remark 1. We would like to take the opportunity to point out some minor errors, which we found in the papers [5, 8]. In [5] the first author claimed that (13) is equivalent to the Turán type inequality [5, Eq. 3.3]

$$
(\nu-1) K_{\nu-1}(u) K_{\nu+1}(u)-(2 \nu-1)\left[K_{\nu}(u)\right]^{2}<0
$$

and conjectured that (16) holds true for all real $\nu \geq 0$. Unfortunately, this claim is not true. We note that (13) is actually equivalent to the Turán type inequality (11). This can be verified by using the recurrence relations (4) and (5) and by using the fact that $K_{\nu}$ is decreasing on $(0, \infty)$ for all $\nu \in \mathbb{R}$. All the same, (16) is valid for all $\nu \in[0,1]$ and $u>0$; this follows from (6), as was pointed out in [8]. Moreover, in [8] the first displayed inequality after Theorem 3.1 should be

$$
K_{\nu}^{2}(u)-K_{\nu-1}(u) K_{\nu+1}(u)<\frac{\nu}{1-\nu} K_{\nu}^{2}(u)
$$

just like before Theorem 3.1 in 8 . This is an equivalent form of (16), and in view of the fact that the constant zero is the best possible in (6) we conclude that for $\nu>1$ the inequality (17) or (16) does not hold. Moreover, the claim in [8] that (11) improves (16), when $\nu>1$, is not true. In view of (17), there is no connection between (11) and (16).

We note that using the symmetry relation $K_{\nu}(u)=K_{-\nu}(u)$, the inequality (16) can be rewritten as

$$
(\nu+1) K_{\nu-1}(u) K_{\nu+1}(u)-(2 \nu+1)\left[K_{\nu}(u)\right]^{2}>0 .
$$

In [5] we conjectured that (18) holds true for all $\nu \leq 0$ and $u>0$. It is important to point out here that in view of the above discussion on (16) the inequality (18) holds true for $\nu \in[-1,0]$, but does not holds for $\nu \leq-1$.

Finally, we note that in the proof of Theorem 3.1 in [8] the explicit formulas for the function $\phi_{\nu}:(0, \infty) \rightarrow \mathbb{R}$, defined by

$$
\phi_{\nu}(u)=1-\frac{K_{\nu-1}(u) K_{\nu+1}(u)}{K_{\nu}^{2}(u)}
$$


and its derivative, should be written as

$$
\begin{aligned}
\phi_{\nu}(u) & =-\frac{1}{u}\left[\frac{4}{\pi^{2}} \int_{0}^{\infty} \frac{u \gamma(t) d t}{u^{2}+t^{2}}+\frac{4}{\pi^{2}} \int_{0}^{\infty} \frac{u\left(t^{2}-u^{2}\right) \gamma(t) d t}{\left(u^{2}+t^{2}\right)^{2}}\right] \\
& =-\frac{4}{\pi^{2}} \int_{0}^{\infty} \frac{2 t^{2} \gamma(t) d t}{\left(u^{2}+t^{2}\right)^{2}},
\end{aligned}
$$

and consequently

$$
\phi_{\nu}^{\prime}(u)=\frac{32}{\pi^{2}} \int_{0}^{\infty} \frac{u t^{2} \gamma(t) d t}{\left(u^{2}+t^{2}\right)^{3}} .
$$

Fortunately, this mistake does not affect the proof of Theorem 3.1 in $[8$. The proof of Turán type inequalities (6) and (11) is correct with the above small modifications.

\section{TURÁN TYPE INEQUALITIES FOR MODIFIED BESSEL FUNCTIONS}

In this section we are going to present some new Turán type inequalities for modified Bessel functions of the first and second kind. As we can see below these inequalities are consequences of some more general results on these functions. Before we state and prove the first main result of this paper let us recall some basics. By definition, a function $f$ is said to be completely monotonic (c.m.) on an interval $\Delta$ if $f$ has derivatives of all orders on $\Delta$ which alternate successively in sign; that is,

$$
(-1)^{n} f^{(n)}(u) \geq 0
$$

for all $u \in \Delta$ and for all $n \geq 0$. It is known that c.m. functions play an eminent role in areas such as probability theory, numerical analysis, physics, and theory of special functions. An interesting exposition of the main results on c.m. functions is given in Widder's book [36] (see also [29]).

The following results complement the discussion given in Section 1.

Theorem 1. Let $I_{\nu}$ and $K_{\nu}$ be the modified Bessel functions of the first and second kind. Then the following assertions are true:

(1) the function $\nu \mapsto I_{\sqrt{\nu}}(u)$ is log-convex on $(0, \infty)$ for all $u>0$ fixed;

(2) the function $\nu \mapsto K_{\sqrt{\nu}}(u)$ is log-concave on $(0, \infty)$ for all $u>0$ fixed;

(3) the function $\nu \mapsto K_{\sqrt{\nu}}(u) / K_{\sqrt{\nu}+1}(u)$ is decreasing on $(0, \infty)$ for all $u>0$ fixed;

(4) the function $\nu \mapsto I_{\sqrt{\nu}}(u) K_{\sqrt{\nu}}(u)$ is log-convex on $(0, \infty)$ for all $u>0$ fixed;

(5) the function $\nu \mapsto I_{\sqrt{\nu}}(u) / K_{\sqrt{\nu}}(u)$ is log-convex on $(0, \infty)$ for all $u>0$ fixed;

(6) the function $\nu \mapsto I_{\nu}(u) / K_{\nu}(u)$ is log-concave on $(-1, \infty)$ for all $u>0$ fixed.

In particular, for all $\nu>0$ and $u>0$, the following Turán type inequalities hold:

$$
\begin{gathered}
I_{\sqrt{\nu+1}}^{2}(u) \leq_{\sqrt{\nu}}(u) I_{\sqrt{\nu+2}}(u), \\
K_{\sqrt{\nu+1}}^{2}(u) \geq K_{\sqrt{\nu}}(u) K_{\sqrt{\nu+2}}(u), \\
K_{\sqrt{\nu+1}}(u) K_{\sqrt{\nu}+1}(u) \leq_{\sqrt{\nu}}(u) K_{\sqrt{\nu+1}+1}(u), \\
I_{\sqrt{\nu+1}}^{2}(u) K_{\sqrt{\nu+1}}^{2}(u) \leq_{\sqrt{\nu}}(u) K_{\sqrt{\nu}}(u) I_{\sqrt{\nu+2}}(u) K_{\sqrt{\nu+2}}(u), \\
I_{\sqrt{\nu+1}}^{2}(u) / K_{\sqrt{\nu+1}}^{2}(u) \leq\left[I_{\sqrt{\nu}}(u) / K_{\sqrt{\nu}}(u)\right]\left[I_{\sqrt{\nu+2}}(u) / K_{\sqrt{\nu+2}}(u)\right],
\end{gathered}
$$

and

$$
I_{\nu+1}^{2}(u) / K_{\nu+1}^{2}(u) \leq\left[I_{\nu}(u) / K_{\nu}(u)\right]\left[I_{\nu+2}(u) / K_{\nu+2}(u)\right]
$$


Proof. For the proof of (1) - (4) we use the following results for modified Bessel functions:

(a) the function $\nu^{2} \mapsto I_{\nu}(u)$ is c.m. on $[0, \infty)$ for each $u>0$ fixed;

(b) the function $\nu^{2} \mapsto 1 / K_{\nu}(u)$ is c.m. on $[0, \infty)$ for each $u>0$ fixed;

(c) the function $\nu \mapsto K_{\sqrt{\nu}+\alpha}(u) / K_{\sqrt{\nu}+\alpha+n}(u)$ is c.m. on $[0, \infty)$ for all $u>0$, $\alpha \geq 0$ and $n \in\{1,2,3, \ldots\}$ fixed;

(d) the function $\nu^{2} \mapsto I_{\nu}(u) K_{\nu}(v)$ is c.m. on $(0, \infty)$ for each $v \geq u>0$ fixed.

Parts (a) and (d) were proved by Hartman and Watson [16], part (b) is due to Hartman [14 and part (c) was obtained by Ismail [21. Now, recall that if the function $f$ is c.m. and the function $g$ is nonnegative with a c.m. derivative, then the composite function $f \circ g$ is also c.m. (see [9, 36]). Thus, since $\nu \mapsto[\sqrt{\nu}]^{\prime}$ is c.m. on $(0, \infty)$, from parts $(\mathrm{a})-(\mathrm{d})$ we obtain the following results:

(i) the function $\nu \mapsto I_{\sqrt{\nu}}(u)$ is c.m. on $(0, \infty)$ for each $u>0$ fixed;

(ii) the function $\nu \mapsto 1 / K_{\sqrt{\nu}}(u)$ is c.m. on $(0, \infty)$ for each $u>0$ fixed;

(iii) the function $\nu \mapsto K_{\sqrt{\nu}}(u) / K_{\sqrt{\nu}+1}(u)$ is c.m. on $(0, \infty)$ for all $u>0$ fixed;

(iv) the function $\nu \mapsto I_{\sqrt{\nu}}(u) K_{\sqrt{\nu}}(u)$ is c.m. on $(0, \infty)$ for each $u>0$ fixed.

On the other hand, each nonnegative c.m. function is log-convex (see [36]), and thus parts (i), (ii) and (iv) imply parts (1), (2) and (4) of this theorem, while part (3) follows from (iii).

We note that actually part (3) can be proved directly by using the formula (see [22])

$$
\frac{K_{\nu-1}(\sqrt{u})}{\sqrt{u} K_{\nu}(\sqrt{u})}=\frac{4}{\pi^{2}} \int_{0}^{\infty} \frac{\gamma_{\nu}(t) d t}{u+t^{2}}, \quad \text { where } \quad \gamma_{\nu}(t)=\frac{t^{-1}}{J_{\nu}^{2}(t)+Y_{\nu}^{2}(t)}
$$

and $u, \nu>0$. Here $J_{\nu}$ and $Y_{\nu}$ stand for the Bessel functions of the first and second kind, respectively. More precisely, in view of the Nicholson formula 35.

$$
J_{\nu}^{2}(u)+Y_{\nu}^{2}(u)=\frac{8}{\pi^{2}} \int_{0}^{\infty} K_{0}(2 u \sinh t) \cosh (2 \nu t) d t
$$

the function $\nu \mapsto \gamma_{\nu}(t)$ is decreasing on $(0, \infty)$ and so is the function $\nu \mapsto \gamma_{\sqrt{\nu}+1}(t)$ for all $t>0$ fixed. This in turn implies that the function

$$
\nu \mapsto \frac{K_{\sqrt{\nu}}(u)}{K_{\sqrt{\nu}+1}(u)}=\frac{4 u}{\pi^{2}} \int_{0}^{\infty} \frac{\gamma_{\sqrt{\nu}+1}(t) d t}{u^{2}+t^{2}}
$$

is decreasing on $(0, \infty)$ for all $u>0$ fixed.

Finally, we note that part (3) can also be obtained directly by using the fact that the function $\nu \mapsto K_{\nu+a}(u) / K_{\nu}(u)$ is increasing on $\mathbb{R}$ for each fixed $u>0$ and $a>0$ (see [23]).

(5) This follows easily from parts (1) and (2) of this theorem.

(6) Similarly, this follows from the facts (see [7]) that $\nu \mapsto I_{\nu}(u)$ is log-concave on $(-1, \infty)$ and $\nu \mapsto K_{\nu}(u)$ is log-convex on $\mathbb{R}$ for all $u>0$ fixed.

\section{Monotonicity properties of the product OF MODIFIED BESSEL FUNCTIONS}

Our second main result reads as follows.

Theorem 2. The function $\nu \mapsto I_{\nu}(u) K_{\nu}(u)$ is decreasing on $(0, \infty)$ for all $u>0$ fixed. Moreover, for all $\nu \geq 1 / 2$ and $u>0$ we have

$$
2 I_{\nu}(u) K_{\nu}(u) \leq I_{\nu-1}(u) K_{\nu-1}(u)+I_{\nu+1}(u) K_{\nu+1}(u) .
$$


In particular, the sequence $\left\{I_{n}(u) K_{n}(u)\right\}_{n \geq 1}$ is decreasing and convex and for all $u>0$ we have

$$
I_{0}(u) K_{0}(u)>I_{1}(u) K_{1}(u)>I_{2}(u) K_{2}(u)>\cdots>I_{n}(u) K_{n}(u)>\cdots .
$$

Proof. Recall that the function $\nu^{2} \mapsto I_{\nu}(u) K_{\nu}(v)$ is c.m. on $(0, \infty)$ for each $v \geq$ $u>0$ fixed (see [16]), and consequently the function $\nu \mapsto I_{\sqrt{\nu}}(u) K_{\sqrt{\nu}}(u)$ is also c.m. on $(0, \infty)$ for each $u>0$ fixed (see part (iv) in the proof of Theorem 10). In particular, the function $\nu \mapsto I_{\sqrt{\nu}}(u) K_{\sqrt{\nu}}(u)$ is decreasing on $(0, \infty)$ for each $u>0$ fixed, and hence the function $\nu \mapsto I_{\nu}(u) K_{\nu}(u)$ is also decreasing on $(0, \infty)$ for all $u>0$ fixed. Thus, for all $u>0$ we have

$$
I_{1}(u) K_{1}(u)>I_{2}(u) K_{2}(u)>\cdots>I_{n}(u) K_{n}(u)>\cdots .
$$

Now, we are going to prove (19). By using the notation $P_{\nu}(u)=I_{\nu}(u) K_{\nu}(u)$, the inequality (19) can be rewritten as

$$
2 P_{\nu}(u) \leq P_{\nu-1}(u)+P_{\nu+1}(u) .
$$

We note that for the function $P_{\nu}$ the following recurrence formulas (see [31]) are valid:

$$
2 \nu P_{\nu}^{\prime}(u)=u\left(P_{\nu+1}(u)-P_{\nu-1}(u)\right)
$$

and

$$
2 \nu P_{\nu}^{\prime \prime}(u)=4 \nu P_{\nu}(u)-(2 \nu-1) P_{\nu-1}(u)-(2 \nu+1) P_{\nu+1}(u) .
$$

On the other hand, owing to Hartman [15], we know that the function $u \mapsto u P_{\nu}(u)$ is concave on $(0, \infty)$ for all $\nu>1 / 2$. Since $u \mapsto 2 u I_{1 / 2}(u) K_{1 / 2}(u)=1-e^{-2 u}$ is concave on $(0, \infty)$, we conclude that in fact the function $u \mapsto u P_{\nu}(u)$ is concave on $(0, \infty)$ for all $\nu \geq 1 / 2$. Consequently, for all $u>0$ and $\nu \geq 1 / 2$ we have

$$
u P_{\nu}^{\prime \prime}(u) \leq-2 P_{\nu}^{\prime}(u)
$$

Now, combining this with (22) and (23) we obtain for all $u>0$ and $\nu \geq 1 / 2$,

$$
2 \nu\left[2 P_{\nu}(u)-\left(P_{\nu-1}(u)+P_{\nu+1}(u)\right)\right] \leq 0,
$$

which is equivalent to (21). Finally, by using (19) for $\nu=1$ we obtain

$$
P_{1}(u)-P_{0}(u) \leq P_{2}(u)-P_{1}(u),
$$

and hence by using the fact that the sequence $\left\{I_{n}(u) K_{n}(u)\right\}_{n \geq 1}$ is decreasing for each $u>0$, it follows that the sequence $\left\{I_{n}(u) K_{n}(u)\right\}_{n \geq 0}$ is also decreasing for each $u>0$; i.e. (20) is valid. With this the proof is complete.

\section{COMMENTS AND CONCLUDING REMARKS}

1. In [12, 18, 19] (see also [17), the authors study the existence, stability and interaction of localized structures in a one-dimensional generalized FitzHugh-Nagumo type model. Recently, van Heijster and Sandstede [20] started to analyze the existence and stability of radially symmetric solutions in the planar variant of this model. The product of modified Bessel functions $I_{\nu}(u) K_{\nu}(u)$ discussed in this note arises naturally in their stability analysis, and the monotonicity condition (20) is important to conclude (in)stability of these radially symmetric solutions. 
After we finished the first draft of this paper, van Heijster informed us that the monotonicity of $\nu \mapsto I_{\nu}(u) K_{\nu}(u)$ is in fact an immediate consequence of the integral formula [28, p. 98]

$$
I_{\nu}(u) K_{\nu}(u)=\int_{0}^{\infty} I_{2 \nu}(2 u \sinh t) e^{-2 u \cosh t} d t
$$

and the monotonicity of $\nu \mapsto I_{\nu}(u)$. More precisely, due to Cochran 11] we know that $\nu \mapsto I_{\nu}(u)$ is strictly decreasing on $[0, \infty)$ for all $u>0$ fixed. By using this result and the above integral formula we conclude that in fact $\nu \mapsto I_{\nu}(u) K_{\nu}(u)$ is strictly decreasing on $[0, \infty)$ for all $u>0$ fixed. Thanks are due to van Heijster for the above information.

2. Let us recall that the function $u \mapsto P_{\nu}(u)=I_{\nu}(u) K_{\nu}(u)$ is strictly decreasing on $(0, \infty)$ for all $\nu>-1$. For $\nu=n \geq 1$, a positive integer, this was proved in 1950 by Phillips and Malin [32, for $\nu \geq 0$ real in 2007 by Penfold et al. 31, and for $\nu \geq-1 / 2$ and $\nu>-1$ recently by the first author [5, 8 . We note that by using (22) and the monotonicity of $\nu \mapsto P_{\nu}(u)$, proved in Theorem 2 we obtain immediately that $u \mapsto P_{\nu}(u)$ is strictly decreasing on $(0, \infty)$ for all $\nu>1$.

Now, let us consider the function $u \mapsto 2 u P_{\nu}(u)$. Hartman and Watson [16] proved that this function is a continuous cumulative distribution function on $(0, \infty)$ for all $\nu \geq 1 / 2$. This function actually maps $(0, \infty)$ into $(0,1)$. Moreover, later Hartman [15] proved that $u \mapsto 2 u P_{\nu}(u)$ is concave on $(0, \infty)$ for all $\nu>1 / 2$, and as we pointed out in the proof of Theorem 2, this result is valid also for $\nu=1 / 2$. We would like to point out here that actually the monotonicity of $u \mapsto P_{\nu}(u)$ for $\nu \geq 1 / 2$ is almost trivial by using the aforementioned concavity result of Hartman. To see this let us recall a particular form of the Pinelis version of the monotone form of l'Hospital's rule (see 33 ):

Let $f, g:(a, b) \subset \mathbb{R} \rightarrow \mathbb{R}$ be differentiable functions on $(a, b)$ with $g^{\prime}(u) \neq 0$ for all $u \in(a, b)$. Furthermore, suppose that $\lim _{u \rightarrow a} f(u)=\lim _{u \rightarrow a} g(u)=0$ and $f^{\prime} / g^{\prime}$ is decreasing on $(a, b)$. Then the ratio $f / g$ is decreasing too on $(a, b)$.

Appealing to this result, since $\lim _{u \rightarrow 0} 2 u P_{\nu}(u)=\lim _{u \rightarrow 0} u / \nu=0$, for $\nu \geq 1 / 2$, to prove the monotonicity of $u \mapsto P_{\nu}(u)=u P_{\nu}(u) / u$ it is enough to show that $u \mapsto\left[u P_{\nu}(u)\right]^{\prime} / u^{\prime}$ is decreasing, which is clearly true because $u \mapsto u P_{\nu}(u)$ is concave.

3. Finally, we note that the product $P_{\nu}$ is useful also in other problems of applied mathematics. For example, in 1986 Cantrell [10] derived tight upper bounds for the function $u \mapsto u I_{n+1}(u) K_{m+1}(u)$, in order to obtain suitable truncation and transient errors in the computation of the generalized Marcum $Q$-function.

Motivated by Theorems 1 and 2 we pose the following problem.

Open problem. Is it true that $\nu \mapsto I_{\nu}(u) K_{\nu}(u)$ is log-convex on $(-1, \infty)$ for all $u>0$ fixed?

\section{ACKNOWLEDGMENTS}

The research of the first author was supported by the János Bolyai Research Scholarship of the Hungarian Academy of Sciences, by the Romanian National Council for Scientific Research in Education CNCSIS-UEFISCSU, project number PN-II-RU-PD_388/2011 and was completed during this author's visit to the Indian Institute of Technology Madras, Chennai, India. The visit was supported by a 
partial travel grant from the Commission on Development and Exchanges, International Mathematical Union. The authors are indebted to Peter van Heijster for his useful comments and suggestions, which enhanced this paper.

\section{REFERENCES}

[1] D.E. Amos, Computation of modified Bessel functions and their ratios, Mathematics of Computation 28 (1974) 239-251. MR0333287 (48:11612)

[2] Á. BARICZ, Turán type inequalities for generalized complete elliptic integrals, Mathematische Zeitschrift 256(4) (2007) 895-911. MR2308896 (2008f:26016)

[3] Á. BAricz, Turán type inequalities for hypergeometric functions, Proceedings of the American Mathematical Society 136(9) (2008) 3223-3229. MR2407087 (2009e:33008)

[4] Á. BARICZ, Functional inequalities involving Bessel and modified Bessel functions of the first kind, Expositiones Mathematicae 26(3) (2008) 279-293. MR.2437098 (2009k:33021)

[5] Á. BAricz, On a product of modified Bessel functions, Proceedings of the American Mathematical Society 137(1) (2009) 189-193. MR2439440(2009i:33005)

[6] Á. BARICZ, Tight bounds for the generalized Marcum Q-function, Journal of Mathematical Analysis and Applications 360(1) (2009) 265-277. MR2548382(2010h:33029)

[7] Á. BARICz, Turán type inequalities for some probability density functions, Studia Scientiarium Mathematicarum Hungarica 47 (2010) 175-189.

[8] Á. BARICz, Turán type inequalities for modified Bessel functions, Bulletin of the Australian Mathematical Society 82 (2010) 254-264. MR2685149 (2011k:33009)

[9] S. Bochner, Harmonic Analysis and the Theory of Probability, Univ. of California Press, Berkeley-Los Angeles, 1960. MR0072370(17:273d)

[10] P.E. Cantrell, On the calculation of the generalized $Q$-function via Parl's method, IEEE Transactions on Information Theory 32(6) (1986) 817-824.

[11] J.A. Cochran, The monotonicity of modified Bessel functions with respect to their order, Journal of Mathematics and Physics 46 (1967) 220-222. MR0213624 (35:4482)

[12] A. Doelman, P. van Heijster and T.J. Kaper, Pulse dynamics in a three-component system: existence analysis, Journal of Dynamics and Differential Equations 21(1) (2009) 73-115. MR2482009(2010d:35176)

[13] T.H. Gronwall, An inequality for the Bessel functions of the first kind with imaginary argument, Annals of Mathematics 33(2) (1932) 275-278. MR1503051

[14] P. Hartman, Completely monotone families of solutions of $n$-th order linear differential equations and infinitely divisible distributions, Annali della Scuola Normale Superiore di Pisa, Classe di Scienze $4^{e}$ série 3(2) (1976) 267-287. MR0404760 (53:8560)

[15] P. Hartman, On the products of solutions of second order disconjugate differential equations and the Whittaker differential equation, SIAM Journal on Mathematical Analysis 8 (1977) 558-571. MR0435510 (55:8469)

[16] P. Hartman and G.S. Watson, "Normal" distribution functions on spheres and the modified Bessel functions, The Annals of Probability 2(4) (1974) 593-607. MR0370687 (51:6913)

[17] P. VAn Heisster, Front Interactions in a Three-Component System, Ph.D. Thesis, Universiteit van Amsterdam, Amsterdam, 2009.

[18] P. van Heijster, A. Doelman and T.J. Kaper, Pulse dynamics in a three-component system: stability and bifurcations, Physica D. Nonlinear Phenomena 237(24) (2008) 33353368. MR2477026(2009j:37124)

[19] P. van Heijster, A. Doelman, T.J. Kaper and K. Promislow, Front interactions in a three-component system, SIAM Journal on Applied Dynamical Systems 9 (2010) 292-332. MR2644922 (2011f:35159)

[20] P. VAn Heisster and B. Sandstede, Planar radial spots in a three-component FitzHughNagumo system, Journal of Nonlinear Science 21(5) (2011) 705-745. MR:2841985

[21] M.E.H. IsmaIL, Integral representations and complete monotonicity of various quotients of Bessel functions, Canadian Journal of Mathematics 29(6) (1977) 1198-1207. MR0463527 $(57: 3474)$

[22] M.E.H. Ismail, Bessel functions and the infinite divisibility of the Student $t$-distribution, The Annals of Probability 5(4) (1977) 582-585. MR0448480 (56:6786) 
[23] M.E.H. Ismail and M.E. Muldoon, Monotonicity of the zeros of a cross-product of Bessel functions, SIAM Journal on Mathematical Analysis 9(4) (1978) 759-767. MR0486686 (58:6388)

[24] C.M. Joshi and S.K. Bissu, Some inequalities of Bessel and modified Bessel functions, Journal of the Australian Mathematical Society (Series A) 50 (1991) 333-342. MR.1094928 (92d:33007)

[25] A. LAFORGia and P. NAtAlini, On some Turán-type inequalities, Journal of Inequalities and Applications (2006) Article 29828, 6 pp. MR2221215 (2007c:26028)

[26] A. Laforgia and P. Natalini, Some inequalities for modified Bessel functions, Journal of Inequalities and Applications (2010) Article 253035, 10 pp. MR2592860 (2011b:26025)

[27] L. Lorch, Monotonicity of the zeros of a cross product of Bessel functions, Methods and Applications of Analysis 1(1) (1994) 75-80. MR1260384 (95b:33008)

[28] W. Magnus, F. Oberhettinger and R.P. Soni, Formulas and theorems for the special functions of mathematical physics, Third enlarged edition. Die Grundlehren der mathematischen Wissenschaften, Band 52, Springer-Verlag New York, Inc., New York, 1966. MR0232968 $(38: 1291)$

[29] K.S. Miller and G. Samko, Completely monotonic functions, Integral Transforms and Special Functions 12 (2001) 389-402. MR.1872377 (2002j:26006)

[30] M.E. Muldoon, Convexity properties of special functions and their zeros, in: G.V. Milovanovic (Ed.), Recent progress in inequalities, Math. Appl. 430, Kluwer Academic Publishers, Dordrecht (1998) 309-323. MR1609967

[31] R. Penfold, J.-M. Vanden-Broeck and S. Grandison, Monotonicity of some modified Bessel function products, Integral Transforms and Special Functions 18(2) (2007) 139-144. MR:2290352(2007m:33015)

[32] R.S. Phillips and H. Malin, Bessel function approximations, American Journal of Mathematics 72 (1950) 407-418. MR0035346 (11:720d)

[33] I. Pinelis, L'Hospital's rules for monotonicity, with applications, Journal of Inequalities in Pure and Applied Mathematics 3(1) (2002) Article 5, 5 pp. (electronic). MR1888920 (2003a:26014)

[34] V.R. Thiruvenkatachar and T.S. Nanjundiah, Inequalities concerning Bessel functions and orthogonal polynomials, Proceedings of the Indian Academy of Sciences. Section A 33 (1951) 373-384. MR0048635 (14:44b)

[35] G.N. Watson, A Treatise on the Theory of Bessel Functions, Cambridge Univ. Press, Cambridge, 1944. MR0010746 (6:64a)

[36] D.V. Widder, The Laplace Transform, Princeton Univ. Press, Princeton, 1941. MR0005923 $(3: 232 \mathrm{~d})$

Department of Economics, Babeş-Bolyai University, Cluj-Napoca 400591, Romania

E-mail address: bariczocsi@yahoo.com

Department of Mathematics, Indian Institute of Technology Madras, Chennai 600036 , INDIA

E-mail address: samy@iitm.ac.in 